\title{
Professional Interpreters Teaching Method Research in Undergraduate Education
}

\author{
Li Yan ${ }^{1}$ \\ ${ }^{1}$ College of Foreign Languages and Cultures, Xiamen University, Xiamen, China. \\ E-mail: liyan_386@163.com
}

\begin{abstract}
To better understand the current situations of Russian interpreters Harbin undergraduate teaching, research and teaching in the future to provide interpreters refer to empirical evidence and data, this research in Harbin four universities in the survey as the main form, its teaching arrangements, teaching materials, teaching content and teaching methods were investigated to obtain firsthand information. Based on the analysis of the main parameters of the investigation, for a variety of problems existing on the Russian interpreters undergraduate teaching made constructive comments.
\end{abstract}

Keywords: interpreting teaching, curriculum, teaching mode

\section{Introduction}

Interpreting refers to a form of expression through oral, will hear the information accurately and quickly convert from one language into another language, and then to transfer and exchange of information communication behavior of the purpose of human cross-cultural, a basic language of communication tools (Mei Deming 2003:14) According to statistics, now Russian general undergraduate institutions in third and fourth grade most of the institutions have set up interpreting classes, generally open 1-2 semester, a total of 36-72 hours 18-36 weeks. Because fewer hours, it is difficult to teach the true sense of the interpreters. All along, the relevant research on teaching Russian interpretation of the few, and most of them kick the tires, but mostly subjective narrative, the fact that the lack of specific data, the system can't fully reveal the inherent law of university teaching Russian interpreters. Therefore, the author of the research group decided on the results of previous studies on the current situation of Russian Undergraduate Teaching interpretation of Heilongjiang Province to do a field study of a sample survey. March 2014 April between our interpretation courses in Harbin four Russian universities conducted a field observation, questionnaires and interviews; the status of Russian Interpreter class Universities in China made a detailed investigation and analysis, and put forward some feasible recommendations.

\subsection{Research purposes}

The main purpose of this study include the following aspects:

(1) Survey of Russian interpreters undergraduate curriculum, teaching methods, teaching content needs and expectations, as well as the evaluation of teaching effectiveness;

(2) Investigation of teachers Russian professional interpreters interpreting the curriculum, teaching methods, understand 
and grasp the teaching content, and evaluation of teaching effectiveness;

(3) Comparison of Russian professional teachers and student evaluation of teaching mode and teaching effect interpretation.

\subsection{Research Methodology}

The survey is divided into three steps:

(1) Collect the professional interpreters curriculum program, qualitative research, and in the light of research and education programs related theoretical results related fields at home and abroad on the basis of the questionnaire is divided into two sections;

(2) Respectively Russian professional teachers and school formal questionnaire and interviews, gathering their feedback on the effectiveness of interpreting curriculum;

(3) Aggregate and questionnaire data and analysis, to draw conclusions.

\section{Description of the questionnaire survey}

\subsection{Survey questionnaire objects}

Our colleges and universities in Heilongjiang province, four Russian Foreign Language Institute conducted a survey of students and faculty, which covers four comprehensive university colleges, three levels of Teachers College, science and engineering colleges, so that the findings can be basically Russian interpreters reflect demand courses at different levels Heilongjiang University.

The questionnaire survey targeted at Russian universities specializing in Heilongjiang Province junior and senior students and teachers, a total of 102 people, including:

(1) Russian junior undergraduate students : Choose two natural classes, a total of 28 people (Heilongjiang University, Harbin Institute of Technology). Started interpreting training acceptable.
(2) Russian undergraduate fourthgraders: Choose four natural classes, a total of 70 people (Kazakh Polytechnic, Harbin Normal University). Interpreters have received training system a semester.

(3) Russian professional teachers: A total of four people (professor, associate professor, lecturer 2).

\subsection{Survey questionnaire issued and recycling}

The survey mainly take interviews and questionnaires to collect the relevant data in two ways.

The returned questionnaires preliminary screening and sorting, excluding invalid questionnaires (e.g. not indicate the categories of persons not to answer related questions, answers selected confusion, etc.), the actual recovery of 93 valid questionnaires, the effective response rate $91 \%$. Details are as follows:

(1) Interpreting Undergraduate: 89 (valid response rate, 90\%).

(2) Teachers Interpretation: 4 parts (valid response rate, 100\%)

In addition, we have the content and design of the questionnaire, the questionnaire part of the problem is not designed to conduct interviews with experts and teachers, and conducted on site interpreting courses observation, this study did beneficial supplement.

\section{Statistical analysis of the data and the questionnaire}

\subsection{Interpreting Curriculum System}

The research module were designed 2 questions.

\subsubsection{Question three}

What do you think should offer courses to aid in interpretation teaching? [Multiple choices]
A. Translation Theory
B. Foreign customs and cultures
C. Foreign customs and cultures
D. Oral training
E. Public Speaking Skills 
F. University general education curriculum

G. Other

Table 1. What kind of course should offer to assist interpretation teaching [multiple choice] (the research object : Student)

\begin{tabular}{|l|l|l|}
\hline Answer & Number of people & $\%$ \\
\hline A & 23 & 9.7 \\
\hline B & 65 & 27.3 \\
\hline C & 48 & 20.2 \\
\hline D & 74 & 31.1 \\
\hline E & 20 & 8.4 \\
\hline F & 4 & 1.7 \\
\hline G & 4 & 1.7 \\
\hline
\end{tabular}

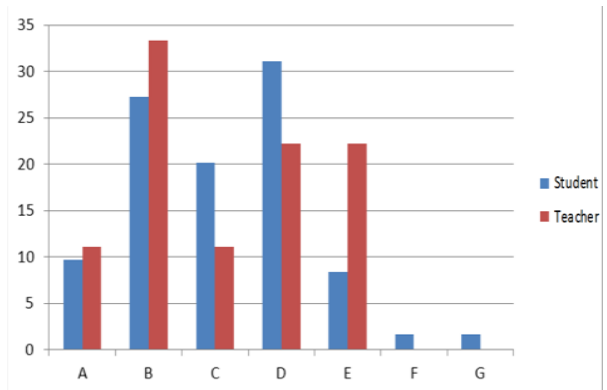

Fig. 1: What kind of course should offer to assist interpretation teaching? (The research object: student teacher)

To open about what courses to aid interpretation teaching, most students and teachers have expressed the need for the creation of "oral training", "listening exercise"; select should offer "translation theory" the highest proportion of students (42.9\%), teachers are all considered to commencement of the course is not open; select the need to open the proportion of students foreign customs and culture" was $20.2 \%, 11.1 \%$ of teachers; Select the creation of "public speaking skills" student ratio of $8.4 \%, 22.2 \%$ of teachers (and select "spoken language training" in the same proportion). According to the statistical results can be found, the teachers and students of the respondents agreed that the biggest obstacle to learning interpretation is speaking and listening level, followed by foreign cultures and speaking skills.

Interpretation faction famous scholar Liu Heping interpretation that the purpose of interpreting lesson is "Professor principles and methods of interpretation, so that students have the interpreters through training and the quality of the basic conditions, namely language skills and verbal skills, good psychological quality, agile reaction, a solid foundation of knowledge, cooperative spirit and work ethic," (Liu Heping 2001). Because just completed undergraduate junior primary stage of language learning, with respect to the level you can learn a language interpreters do not have a strict entry criteria, some of the students at the beginning of interpreting learning level is still a great lack of bilingual, so juniors languages capacity factors influence. To strengthen this aspect of training is necessary to pay attention to student's sense of language. To train student's sense of language ability in interpretation, we can interpret the use of live teaching practice in interpreting around translation, interpretation and other flexible training methods of interpretation.

Respondents student feedback: about Russian culture courses limited availability, content more monotonous, the novelty of the proposed increase in the textbook, you can consider using the original books and audio visual materials. Humanities courses are an important part of teaching aid interpretation, its crosscultural benefit a lot, so we should continue to maintain the novelty and the era of humanities courses, so as our interpreter training services.

\subsubsection{Question 4}

How do you assess the current use of the textbook? [Multiple choices]

A. Good theory with practice

B. The theory part is good, but too difficult practical part 
C. The Theory and Practice of touch, lack of systematic theoretical knowledge

D. Stale writing system, the content of aging, from the actual needs

E. Difficult to evaluate

\section{F. Other}

Table 2. How do you assess the current use of the textbook? (The research object: Student)

\begin{tabular}{|l|l|l|}
\hline Answer & Number of people & $\%$ \\
\hline A & 16 & 17.2 \\
\hline B & 25 & 26.9 \\
\hline C & 17 & 18.3 \\
\hline D & 19 & 20.4 \\
\hline E & 11 & 11.8 \\
\hline F & 5 & 5.4 \\
\hline
\end{tabular}

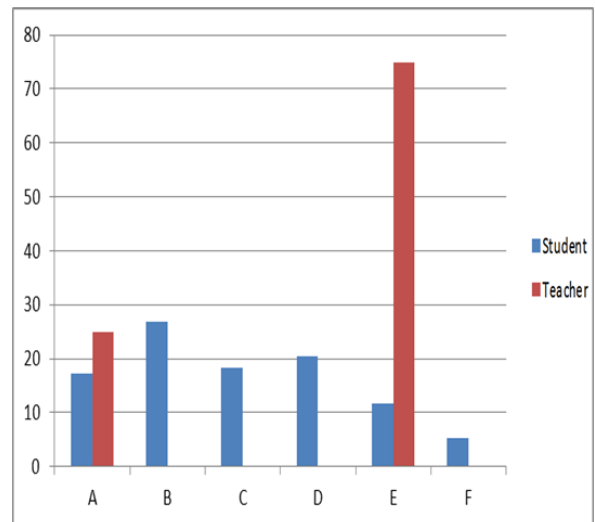

Fig. 2: How do you assess the current use of textbooks? (The research object: student teacher)

In the four universities in this research, only a college textbook on the use of fixed domestic market, the teacher of other institutions have expressed material mainly used for teaching materials from a variety of ways to collect themselves, ranging from newspapers, TV material on the Internet and other media chosen, pick from other published materials in interpreting the material themselves or others to attend practice interpreting the resulting material. The survey results showed that students evaluate teaching materials currently used in a variety of quite proportion, no significant difference. In the interview also has student teachers to reflect material too casual, no system, or too difficult.

\subsection{Interpreting teaching mode and teaching methods}

This module is the focus of research by our group, 2 questions were designed.

\subsubsection{Question 1}

Do you think the main mode of interpretation of classroom teaching should be? [Multiple choices]

A. Professor teacher based, students listen carefully mode

B. To complete a specific task oriented task-based translation model

C. Simulate the actual translation situations to solve practical problems mainly situational mode

D. Under the guidance of teachers, students' independent, cooperative learning based model

E. Student exercises and classroom based site assessment model comment

F. Under the guidance of a teacher, teacher-student interaction patterns

G. Other

Table 3. Main mode of interpreting classroom (survey: student)

\begin{tabular}{|l|l|l|}
\hline Answer & Number of people & $\%$ \\
\hline A & 10 & 4.5 \\
\hline B & 35 & 15.9 \\
\hline C & 63 & 28.6 \\
\hline D & 50 & 22.7 \\
\hline E & 28 & 12.7 \\
\hline F & 34 & 15.5 \\
\hline G & 0 & 0 \\
\hline
\end{tabular}

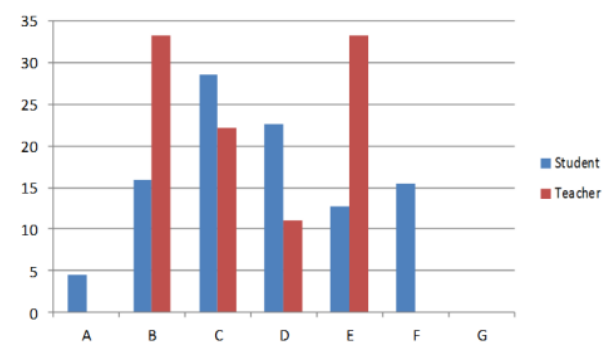


Fig. 3: Main mode of interpreting classroom (The research object: student teacher)

The survey respondents believe that the main mode of interpreting student classroom teaching should be the first to simulate the actual translation situations, in order to solve practical problems mainly situational model (28.6\%), followed by the teacher's guidance, students' independent, cooperative learning based model. B, F, E considerable proportion of the options. The proportion of respondents selecting teachers to complete a specific task oriented task-based translation model and student exercises and classroom based site assessment mode commentary highest (average $33.3 \%$ ), second is to simulate the actual translation situations, in order to solve practical problems mainly situational mode, and under the guidance of teachers, students' independent, cooperative learning based model. In general, task-based, situational, autonomous learning and on site assessment model students and teachers have been given recognition.

\subsubsection{Question 2}

What do you think the biggest problem in interpreting extracurricular training process will encounter? [Multiple choices]

A. Insufficient language skills

B. Interpretation skilled enough to master skills

C. Can't find moderate difficulty training materials

D. No good interpretation training equipment and the environment

E. Missing teacher assessment guidance

F. Other

Table 4. The biggest problem in interpreting extracurricular training process encountered (survey: student)

\begin{tabular}{|l|l|l|}
\hline Answer & Number of people & $\%$ \\
\hline A & 37 & 38.9 \\
\hline B & 21 & 22.1 \\
\hline C & 9 & 9.5 \\
\hline
\end{tabular}

\begin{tabular}{|l|l|l|}
\hline $\mathrm{D}$ & 23 & 24.2 \\
\hline $\mathrm{E}$ & 5 & 5.3 \\
\hline $\mathrm{F}$ & 0 & 0 \\
\hline
\end{tabular}

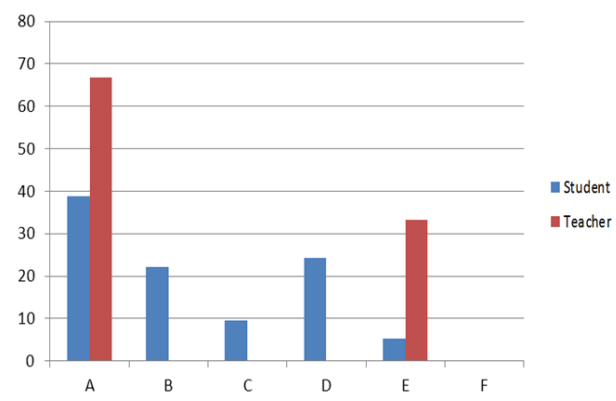

Fig. 4: The biggest problem in interpreting extracurricular training process encountered (The research object: student teacher)

Secondly, the students believe the difficulties of interpretation extracurricular training also includes a "skilled enough to master skills of interpretation" $(22.1 \%)$ and "not a good interpreting training equipment and the environment" (24.2\%); while teachers are considered to be "missing teacher evaluation guide" (33.3\%).

According to the survey results of the main factors affecting the analysis results interpretation course learning outcomes reflected in:

(1) Language skills. Interpreting courses are usually opened in the third year under the semester, the students' basic examination by Russian majors. After two semesters of study, students in the discussion, saying higher frequency of occurrence of "small vocabulary", "grammar is weak," "unfamiliar with the theme," and so on. Student's ability to learn language interpreters should have the basic quality of interpretation in practice the first to be tested, and its lack of interpreters affect learning courses for all.

(2) The ability of skills. Skills include the ability to interpret the required specialized capabilities, as well as the 
general ability of interpretation activities, such as memory, thinking, imagination, attention, etc., for example, notes capability, digital conversion capability, public speaking skills, coping strategies. Student discussion in higher frequency of occurrence another argument is "do not understand", "do not understand", "remember", "inability to concentrate", and other phrases. Frequency of occurrence of such claims can be explained by the course of study the student ability level of skill acquisition level.

This shows that for some students, their learning objective factors produced can't be ignored. Overall, the impact of the course learning outcomes interpretation of objective factors, including software and hardware conditions. Software condition involves two aspects of teachers and teaching. Including teachers teaching style, teaching methods, curriculum and syllabus, teaching objectives, teaching schedule, assessment methods. Among them, the teacher is the key factor, teachers with practical experience of using their own interpretation of experience can give students the most direct inspiration. Hardware requirements include a language laboratory equipment, interpreting teaching, interpreting audio and video library, internet aided teaching, interpreting the real scene simulation.

\section{Conclusion}

On the basis of previous research on the activities of the survey questionnaire basic grasp of courses taught in the case of professional interpreters Russian Heilongjiang Province, to further strengthen the professional Russian interpreter course, systematic construction provides a richer, more objective data. Of course, given the complexity of interpreting events, the survey could not cover all aspects of interpreting course, the need for specialized empirical research and discussion. In addition, due to the limitations in the scope of the investigation, staff representation and other aspects of the data provided by this survey and the corresponding conclusion is just a basic preparatory work, both need to be analyzed and validated by empirical research, but also interpreters need to compare each other and actual observations, more needs in a wider context, the organization deeper investigation in order to obtain a more comprehensive and systematic data, it may be the relationship between memory and the interpretation of a more scientific knowledge and understanding, thus further promote the development of interpretation of cognitive research.

\section{Acknowledgement}

This research topic is sponsored by Fujian Social Sciences Planning Program (No. 2013C082), Constructing the Test System of Professional Certification in Interpretation, and National Social Science Fund: Constructing and Expanding Translational Writing Studies (No. 13BYY043), Xiamen University Fund for Prospering Philosophy and Social Science (No. 0650-Y07200)

\section{References}

[1] Liu Heping interpreters and interpreting scientific thinking skills -- Reasoning Approach [ M ]. Beijing: China Translation and Publishing Corporation, 2001.

[2] Mei Deming to overcome the " interpretation " exam last hurdle : Interpreting $[\mathrm{J}]$ talent and employment, 2003, (19) : 14 to 15. 